\title{
Materialbeschaffung online
}

Fünf Praxen mit 8 Zahnärzten haben sich im süddeutschen Raum unter dentalsynoptics e. V., wissenschaftliche Vereinigung von Zahnärzten, zusammengeschlossen und ein eigenes, computergestütztes QM-System mit Namen PMS (Praxismanagementsystem) entwickelt. Zielvorgabe bei der Entwicklung war die praxisgerechte Umsetzung der gesetzlichen Auflagen, beim Prozess Hygiene unter besonderer Berücksichtigung der RKI-Richtlinien. Entstanden ist unter anderem ein ScannerBarcode-basiertes Hygienesystem, das die gesetzlichen Anforderungen an zahnärztliche Hygiene übertrifft und die Aufbereitung von Geräten und Instrumentarien in allen Funktionsbereichen sicherstellt. Die Zahnärzte der Gemeinschaftspraxis Dres. Altmann in Langenargen am Bodensee sind Mitglieder von dentalsynoptics e. V. und haben das PMS in den Praxisalltag integriert. Ein erfolgreiches Qualitätsmanagement zeichnet sich durch effiziente Arbeitsabläufe aus. Materialbeschaffung über Online-Shops tragen dazu bei, die Materialwirtschaft transparent und übersichtlich zu gestalten. Der Einbezug der für den Hygieneprozess ohnehin vorhandenen Scanner bietet zusätzliche Vorteile. Was bedeutet die Umstellung von Karteikartensystem auf Online-Bestellung für die Praxis und für die damit beauftragte Helferin Fabienne Uhlemair?

Herr Dr. Altmann, beschreiben Sie bitte Ihre Praxisgemeinschaft, damit wir einen Eindruck davon bekommen, in welchem Umfang und zu welchen Fachbereichen Sie Produkte auswählen! Dr. M. Altmann: Meine Kollegen von dentalsynoptics e.V. und ich sind während unserer universitären Ausbildung in Freiburg von Prof. Dr. Jörg Strub geprägt worden und folgen dem synoptischen Behandlungskonzept, d.h. wir streben eine wissenschaftlich fundierte, sorgfältige und individuell konzipierte Behandlungsplanung und Durchführung an. Unser Behandlungsspektrum umfasst nahezu die gesamte moderne Zahnmedizin, und wir müssen für unsere 6 Behandlungszimmer Material für die Bereiche Prophylaxe, konservierende Zahnheilkunde, Endodontie, Prothetik, aber auch für chirurgische Eingriffe einschließlich der Implantologie beschaffen. Das breite Leistungsspektrum zieht ein großes Produktportfolio nach sich. Um den Überblick zu behalten, nutzen wir unsere Ressourcen aus der Verwaltung der Hygieneketten mit Barcodes und Scanner. Zudem bestellen wir fast ausschließlich auf Online-Portalen der jeweiligen Fachfirmen. Hier ist unsere Mitarbeiterin Frau Uhlemair gefragt, die seit 3 Jahren für die Bevorratung der Praxis verantwortlich ist.

Frau Uhlemair, es sind vor allem Verbrauchsgegenstände und rotieren- de Instrumente, die in regelmäßigen Zyklen erneut bestellt werden müssen. Ziehen wir als Beispiel die Marke Komet mit einem professionellen Web-Shop heran. Nennen Sie die Gründe, warum Sie den Web-Shop überhaupt nutzen! F. Uhlemair: Wir sind Anfang 2010 konsequent auf die Online-Bestellung umgestiegen und arbeiten seit 2011 mit dem Komet-Web-Shop. Die Vorteile liegen für mich klar auf der Hand: Ich kann im Internet zeitunabhängig bestellen und arbeite papierlos. Insgesamt fühle ich mich viel flexibler, und der Bestellvorgang läuft schneller ab. Besonders praktisch finde ich die Bestellhistorie, also die Order der letzten Male, an der ich mich immer wieder orientieren kann und die mir Aufschlüsse über die notwendigen Bestellmengen gibt.

Wie haben Sie die Anmeldeformalitäten für den Zugang zum Shop empfunden? Und wie organisieren Sie die Materialverwaltung?

F. Uhlemair: Viele Kolleginnen haben vielleicht Respekt davor, aber der Login ist denkbar einfach und unkompliziert: Kundennummer und Passwort eingeben - fertig! Ich bestelle einmal in der Woche, d.h. zuerst kontrolliere ich die Materialbestände und gleiche die Bestände mit unserer Bestellliste ab. Dann wechsle ich zum Computer und gebe die Artikel in die Order ein. Neben diesen praktischen As- pekten macht mir die Arbeit per Internet viel mehr Spaß als das Ausfüllen von Karteikarten!

Komet praktiziert den Direktvertrieb, d.h. die Betreuung erfolgt über einen persönlichen Komet-Fachberater. Nun erleben wir im Dentalmarkt die Tendenz zu immer erklärungsbedürftigeren Produkten. Wann sind Ihrer Meinung nach die Möglichkeiten der Online-Shops erschöpft?

Dr. M. Altmann: Auf den Komet-Fachberater kann man trotz des Web-Shops nicht verzichten. Der Grund dafür ist einfach: Die Online-Hilfe des Shops kann vieles erläutern, aber wir werden dadurch nicht in die Lage versetzt, das Produkt physisch zu spüren. Einen neuen Kunststoffpolierer oder eine neue Fräse möchte ich in den Händen gehalten haben, bevor ich bestelle.

F. Uhlemair: Produkte, die wir bereits kennen, bestellen wir dann über den Shop.

Dr. M. Altmann: Außerdem hat sich über die Jahre ein vertrauensvolles Verhältnis zwischen dem Fachberater und uns entwickelt und wir wissen: Er kennt uns und das Profil unserer Praxis und weiß, was uns interessieren könnte. Das Angebot des Shops und der regelmäßige, persönliche Besuch des Fachberaters ergänzen sich aus unserer Sicht perfekt.

Wie halten Sie sich über Produktneuheiten auf dem Laufenden?

Dr. M. Altmann: Ich darf unser Praxisteam als sehr fortbildungsrege bezeichnen, und wir bringen von den Fortbildungsveranstaltungen und Messen viele Anregungen mit. Die bereits angesprochene persönliche Betreuung durch den Fachberater, der uns auf interessante firmenspezifische Neuerungen aufmerksam macht, ist aber eine weitere wichtige Säule für die Deckung unseres Informationsbedarfs.

Macht die Online-Bestellung nur für große oder für alle Praxen Sinn? Dr. M. Altmann: Grundsätzlich macht die Online-Bestellung für alle Praxen Sinn. Gerade kleine Praxen müssen in Zukunft die 


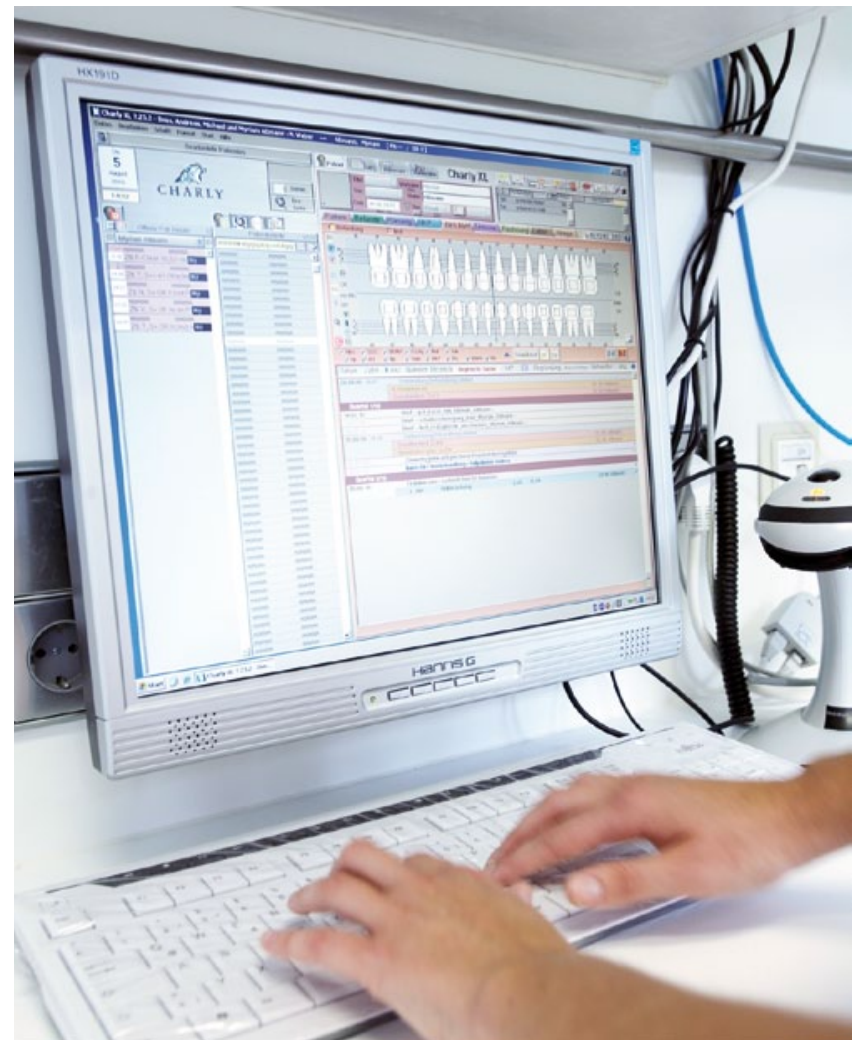

Der Vertrieb bei Komet wird durch den Webshop noch direkter und ist eine Option für die zeitunabhängige Bestellung.

Dr. Andreas Altmann, Myriam und Dr. Michael Altmann (Mitglieder von dentalsynoptics e. V.) und das Praxisteam.

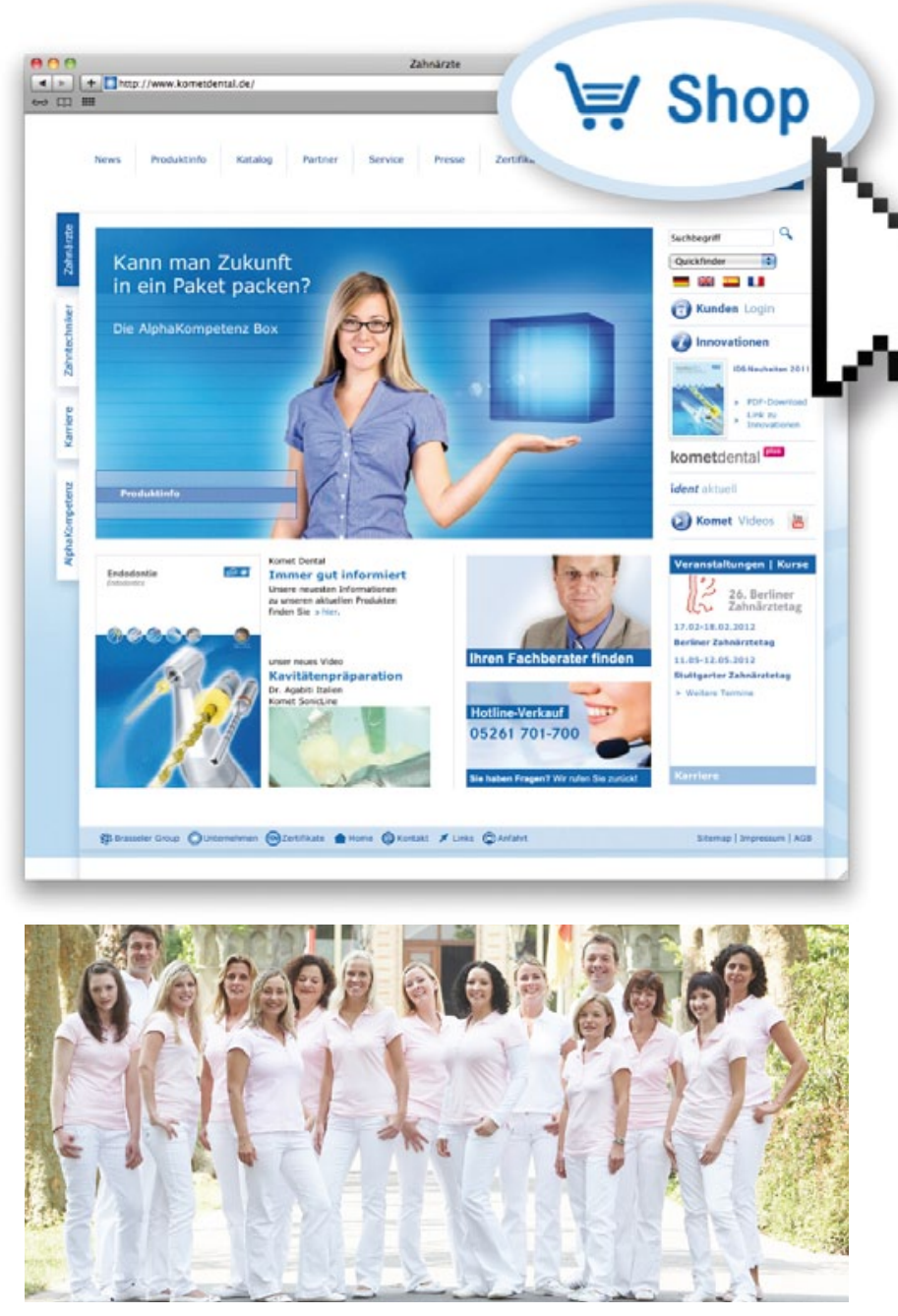

auf dem Behandlungsblatt eines jeden Patienten notieren.

Dr. M. Altmann: Gerade deshalb wünschten wir uns einen engeren Schulterschluss zwischen der Dentalindustrie und den Softwarehäusern, damit die Praxen die gesetzlichen Auflagen und die Pflicht zur Dokumentation mit möglichst wenig Arbeitsaufwand umsetzen können. Mit dentalsynoptics e.V. feilen wir an einer praxisgerechten Umsetzung der Arbeitsabläufe. Bestellungen über WebShops stellen für die Kollegen daher eine einfache, aber effektive Möglichkeit dar, die Vorteile der digitalen Welt für ihre Praxen zu nutzen.

F. Uhlemair: Wir behelfen uns deshalb folgendermaßen: Ich lese die erhaltene Ware ein, drucke dann eigene Barcodes aus und klebe diese auf die Schachteln. Ansonsten müssten wir - noch umständlicher! - auf 2 Ebenen (Hersteller/interner Barcode) dokumentieren. Das klingt nach sehr viel Aufwand, aber man muss sich vor Augen halten, wie wir früher dokumentiert haben: Da musste ich die Ident-Nummern der Produkte auf Karteikarten übertragen und diese dann wieder

\section{! Vielen Dank für das Gespräch.}

Das Interview führte Dorothee Holsten.

\section{Kontakt}

Zahnärztliche Gemeinschaftspraxis

Dr. Andreas Altmann, Myriam Altmann, Dr. Michael Altmann

Mühlstraße 25

88085 Langenargen

E-Mail: praxis@draltmann.de 


\section{Der Goldstandard des Zähneputzens}

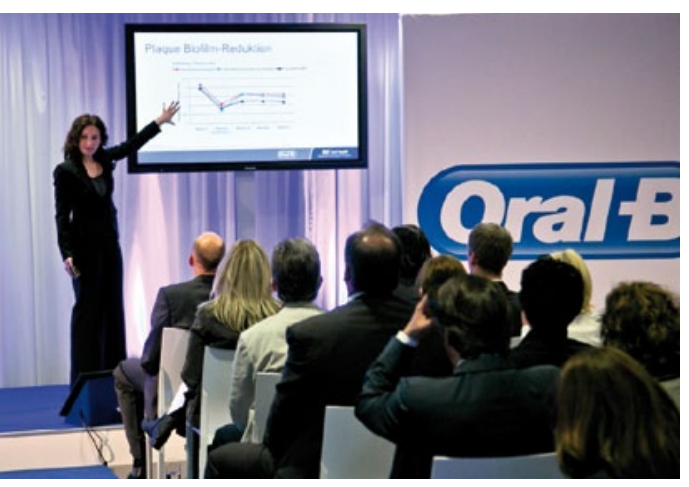

Am 20. April 2012 fand das 4. Oral-B Symposium im Berliner Velodrom statt. Im Vorfeld dieser Veranstaltung wurden am Vormittag zahnmedizinische Wissenschaftler sowie Journalisten von führenden Procter \&Gamble Professional Oral Health-Mitarbeitern über neueste Entwicklungen bei elektrischen Zahnbürsten informiert. Im Zentrum der Vorträge stand die aktuelle Produktinnovation: die neue Oral-B TriZone.
Unter anderem ging Dr. Eva Kaiser, Forschungs- und Entwicklungsabteilung, Produktforschung P\&G Kronberg, in ihrer Präsentation auf die bestehenden Zahnputzgewohnheiten der Benutzer von Handzahnbürsten ein. Sie zählte einige der Gründe auf, warum manche Benutzer die Handzahnbürste bevorzugten und bisher nicht auf die elektrische Zahnpflege umgestiegen seien: So würden einige eigentlich gerne mit einer Elektrozahnbürste von Oral-B putzen, aber den kleinen Oral-B-typischen Bürstenkopf nicht mögen. „Dieser Anwenderkreis bekommt jetzt eine Alternative: die neue Oral-B TriZone“, verkündete Dr. Kaiser. Sie erklärte, die Oral-B TriZone verfüge über 3 Reinigungszonen: Es gäbe einen beweglichen, abgewinkelten Power-Tip zur effizienten Reinigung der schwer erreichbaren Stellen im Mund, beispielsweise der Molaren. Neben einem stationären Borstenfeld mit blauen pulsierendfeststehenden Borstenreihen zur effizien- ten Reinigung der Zahnoberflächen verfüge die Bürste zudem über ein bewegliches Borstenfeld, welches mit seinen weißen pulsierend-schwingenden Borsten tief in die interproximalen Bereiche eindringen könne. „Die Ergebnisse unserer umfangreichen Verbraucherstudien haben klar gezeigt, dass die neue Trizone von ihren Benutzern als hervorragend bewertet wurde“, fasste Dr. Kaiser zusammen. Die Anwender hätten sie ähnlich angenehm wie ihre bisherige Handzahnbürste empfunden. „Eine Änderung der angestammten Putzgewohnheiten ist nicht erforderlich. Besonderer Vorteil der Oral-B TriZone: Es wird bis zu 100\% mehr Plaque entfernt als mit einer herkömmlichen Handzahnbürste.“

Mehr Informationen über die Inhalte der Veranstaltung gibt es online unter www. oralbsymposium.de

Nach Informationen der

Procter \& Gamble Professional Oral Health, Schwalbach im Taunus

\section{Konisch oder Flach}

Für den Kongress Anfang Mai hatte sich das Unternehmen wieder ein klassisches schweizerisches Umfeld gesucht. Das geschichtsträchtige Luzern bot eine ideale Plattform für das diesjährige Motto „feel the puls of science in the heart of Switzerland“. In 7 Sessions diskutierten internationale Referenten und 1300 Gäste aus dem Inn- und Ausland über die aktuellen Themen in der Implantologie. Die Implantat/ Abutment-Verbindung und die klinischen Erfahrungen mit Platform-Switching gaben in den ersten beiden Sessions den richtigen Einstieg in die Veranstaltung. Welche Abutment-Verbindung, flache oder konische, zeigt den besten Langzeiterfolg? Evidenzgestützte Aussagen sind hier widersprüchlich. Für beide Verbindungen gibt es Vor- und Nachteile. Auch über die Dichtigkeit sind die Aussagen nicht einheitlich. Beide Verbindungen zeigen eine gewisse Verformung bei Belastung und somit die Gefahr einer möglichen periimplantären
Infektion. Zu diesem Problemkreis gehört auch die Rotationssicherheit der Verbindungen. Sehr interessant dazu sind die Untersuchungen aus der Physik, dass die rechnerisch vorgegebenen Standardwerte zwar theoretisch auch in der Produktion umgesetzt werden könnten, zumeist aber nicht erreicht werden. In einer Vergleichsstudie, zeigte sich aber, dass die CAMLOG/CONNELOG SCREW-LINE die größte Annäherung an die Idealvorgaben erreicht. Eindringlich wurde hier auch vor den Kopien gewarnt, die in keinem Fall irgendeinem Qualitätsstandard genügen. In diesem Zusammenhang wurde auch die bakterielle Besiedelung des Implantinnenraums angesprochen, die bereits bei der 1. Eröffnung stattfindet. Allerdings entspricht diese Flora nicht unbedingt auch den Keimen, die bei einer Periimplantitis gefunden werden. $\mathrm{Zu}$ beachten ist, dass jede Manipulation an der Abutment-Verbindung (z.B. Auswechseln der Abdeckschraube) die inflammatorische
Wirkung auf das Weichgewebe erhöht. Um dieses Risiko zu vermindern, erscheint das Plattform-Switching (durch kleinere Abutment-Durchmesser wird die Schulterauflage verbreitert und der Abutment-Spalt zur Mitte hin verlagert) gegenüber dem bündigen Abschluss im Vorteil zu sein. Dazu wurden die Möglichkeiten beschrieben, die die beiden Systeme (CAMLOG flache, CONNELOG - konische Verbindung) für den Behandler und Patienten bieten. Je nach chirurgischem Vorgehen (supra-, epioder leicht subcrestal) decken die beiden Systeme alle Indikationen ab. Weitere Themen galten dem alten Patienten sowie den digitalen Möglichkeiten. Ein besonderes Highlight erwartete die Teilnehmer bei der Abendveranstaltung. Nach einer Schifffahrt über den Vierwaldstätter See ging es mit Europas ältester Zahnradbahn $1600 \mathrm{~m}$ auf die Rigi, „die Königin der Berge“, wo uns ein Schweizer Abend in allerschönster Tradition erwartete. 